\title{
INOVAÇÃO EM PROCESSOS DE TRABALHO DE EQUIPES VIRTUAIS: ESTUDO DE CASO DA IMPLANTAÇÃO DE FERRAMENTA DE AUTOMATIZAÇÃO DE FLUXO
}

Larissa Conegundes Moreira (larissaconegundes03@gmail.com) - Pontifícia Universidade Católica de Minas Gerais, PUC-MG.

Lucio Otavio Xavier Moreira (xavierotavio@ hotmail.com) - Pontifícia Universidade Católica de Minas Gerais, PUC-MG.

Nathalia Nathielle da Silva Dimas (nathaliadimas@outlook.com) - Pontifícia Universidade Católica de Minas Gerais, PUC-MG.

Richard Batista da Costa (richard.bcosta@ hotmail.com) - Pontifícia Universidade Católica de Minas Gerais, PUC-MG.

Matheus Luiz Pontelo de Souza (matheusluiz@pucminas.br) - Pontifícia Universidade Católica de Minas Gerais, PUC-MG.

\section{RESUMO}

A pressão por inovar em processos de trabalho, com ênfase à digitalização de tais processos, não é um fenômeno recente. Contudo, foi intensificada devido à pandemia do COVID-19 e à consequente adoção do regime de trabalho remoto. Nesse contexto, ferramentas digitais podem auxiliar no enfrentamento de desafios ligados à comunicação, acompanhamento de atividades, dentre outros. Este artigo apresenta um estudo de casos múltiplos de um processo de implantação de uma ferramenta de automatização de fluxos em três setores de uma empresa. Como resultado pôde-se descrever o processo de implantação da ferramenta, as melhorias geradas no fluxo de trabalho, lições aprendidas e desafios. Em geral, houve aceitação dos envolvidos, e uma recusa pautada no desempenho da equipe mediante a implantação do novo processo, o que possibilitou a percepção da importância de analisar o impacto da inclusão de ferramentas semelhantes na rotina das equipes virtuais.

Palavras-chave: automatização de processos; equipes de trabalho virtual; inovação; ferramentas; pipefy. 


\section{INTRODUÇÃO}

A crise gerada pela pandemia de COVID-19 refletiu em todos os setores da sociedade, deixando um rastro de perdas sem precedentes. Contudo, espera-se que um cenário de reinvenção e aprendizado sucederá à crise. Assim, novos modos de operação ficarão marcados como práticas de sucesso no mercado de trabalho e em outros setores da sociedade. Uma delas é o regime de trabalho remoto, também chamado de home office, no qual os colaboradores trabalham de sua residência.

Contudo, é fato que as empresas já sofram, a pressão por inovar em processos de trabalho. Essa pressão por inovar foi rapidamente catalisada e atingiu grandes proporções com a exigência do isolamento social por parte de entidades governamentais e de saúde. Desafios como promover a comunicação, agir antecipadamente para prever e evitar erros e monitorar as tarefas para que dessa forma a equipe alcance objetivos, fazem parte do cotidiano de uma organização, e a utilização de ferramentas digitais de apoio favorecem o enfrentamento desses desafios.

Muitas dessas ferramentas voltadas ao compartilhamento de informações inerentes aos fluxos operacionais de trabalho afetam áreas afins à gestão, como setores administrativos, financeiros, dentre outros. Portanto, diante da necessidade de evitar retrabalhos e otimizar processos, que se intensificam com o atual cenário de pandemia, é importante entender como as ferramentas auxiliam na automatização de tarefas, e os problemas e aprendizados relacionados a implantação dessas ferramentas. Além disso, estudar conceitos e aplicações de ferramentas é essencial para melhoria de performance de uma empresa.

A partir do cenário exposto, propõe-se como problemática: quais aprendizados e desafios estão envolvidos na implantação de ferramenta de automatização de processos em equipes virtuais? Questões adicionais voltadas às melhores práticas e desafios enfrentados foram exploradas.

Assim sendo, este estudo de caso teve como objetivo descrever o processo de implantação de ferramenta voltada para automatização de processos de trabalho em regime de home office, levantando desafios, lições aprendidas e outros itens que se mostraram relevantes durante a análise. Para isso, foi feito um estudo de casos múltiplos nos setores Financeiro, Comercial e Recursos Humanos de uma empresa com cerca de 300 funcionários e que desenvolve Software de Gestão Comportamental de Pessoas. 


\section{REVISÃO TEÓRICA}

\subsection{Fluxo de trabalho, informação e trabalho remoto}

Souza (2003) aborda que fluxo de trabalho pode ser entendido como qualquer tarefa executada por dois ou mais membros de um grupo de trabalho visando um objetivo comum. Uma variação dos fluxos de trabalho compreende modelos computadorizados de processos que especificam os parâmetros envolvidos em sua execução, para permitir o trabalho integrado e interativo em grupo.

De acordo com Valentim e Zwaretch (2006) os fluxos de informação são orientados, entre outros aspectos, pela e para a comunicação organizacional, sendo compreendidos como resultados do processo de interação entre as pessoas de um ambiente organizacional. Assim como abordam Sugahara, Jannuzzi e Falsarella (2015), as organizações podem explicitar a existência da sua cultura e confiança entre as pessoas e ideias quando compartilham as informações.

Contudo, a recente mudança para o trabalho em ambiente virtual, especialmente devido à pandemia do COVID-19, gerou significativo impacto e novos desafios para as equipes virtuais de trabalho em termos da gestão efetiva de seus processos de trabalho, especialmente no que tange às dinâmicas de comunicação e colaboração (ABARCA; PALOS-SANCHEZ; RUSARIAS, 2020).

Segundo Olariu e Aldea (2014), a definição de equipe virtual mais aceita é a de um grupo de trabalhadores dispersos pela geografia, organização ou pelo tempo, reunidos por tecnologias de informação para realizar tarefas organizacionais. Assim, o principal fator de coordenação do trabalho em equipes virtuais são as tecnologias de apoio à comunicação (ABARCA; PALOS-SANCHEZ; RUS-ARIAS, 2020; HERTEL; GEISTER; KONRADT, 2005).

Inúmeras são as tecnologias desenvolvidas que possibilitam a troca de informação e comunicação dos membros de uma equipe, permitindo uma colaboração eficaz, inclusive em um regime remoto. Como abordam Abarca, Palos-Sanchez e Rus-Arias (2020), o trabalho remoto é definido como trabalho realizado longe da sede da empresa ou da fábrica, no qual o trabalhador não tem contato pessoal com outros colegas de trabalho, mas é capaz de comunicar com eles utilizando tecnologia moderna. 
A comunicação em uma equipe virtual é um tema-chave consideravelmente investigado, sendo explorados aspectos como diversidade cultural, distribuição da equipe, e a liderança enquanto fatores que influenciam o trabalho virtual (ABARCA; PALOS-SANCHEZ; RUSARIAS, 2020).

Administrar equipes virtuais é um desafio, mesmo para quem considera a equipe virtual como derivada da equipe tradicional. Assim a criação de equipes virtuais apresenta razões estratégicas, cita-se a capacidade do capital humano, acesso aos profissionais do mundo todo, adaptabilidade e outros (POLETE et al., 2012).

\subsection{Gestão e automatização de processos e suas ferramentas de apoio}

Processos são um conjunto sequencial e finito de tarefas que devem ser executadas para transformar algum insumo/entrada em algo útil, uma saída, atendendo especificações definidas e sendo executado por pessoas ou máquinas (WILDAUER, 2015). Segundo Lage Júnior (2016), a gestão por processos visa que os agentes interajam entre si e com o meio, em uma relação de interdependência enfatizando a importância das atividades integradas que compõem os processos de negócios e aos fluxos de informação.

Mattos (2012) enfatiza o papel da tecnologia de informação na gestão, para facilitar o planejamento e implementação de novas capacidades tecnológicas e otimização de processos. Zorgios e Karakostas (2008) dizem que a automatização de processos, em específico para a gestão de processos de negócios e gestão de fluxos de trabalho, pode ser definida como o uso de tecnologias da informação para automatizar total ou parcialmente a execução e gestão de processos de negócio.

Várias são as ferramentas tecnológicas desenvolvidas para auxiliar as pessoas e organizações em suas atividades umas delas sendo a ferramenta para o gerenciamento de atividades Pipefy. Segundo Cardoso (2019), o Pipefy pode ser considerado como um software de gerenciamento empresarial e de feedbacks dos colaboradores, com uma interface intuitiva, cores chamativas e outras ferramentas.

Segundo Oliveira (2020), o Pipefy, por ser baseado em nuvem, em que não precisa instalar nenhum software para usá-lo, o que, junto ao conjunto de ferramentas de gerenciamento disponíveis, permite que se adote o tipo de gestão e automação precisos para o desenvolvimento de um fluxo de trabalho eficaz ou para o planejamento do dia/semana/mês da equipe. 
O Pipefy traz a visualização em lista e em formato Kanban, que facilita a visualização dos pipes em estágios, conforme Figura 1. Além disso, não requer conhecimento técnico, pois possui uma interface gráfica fácil de usar.

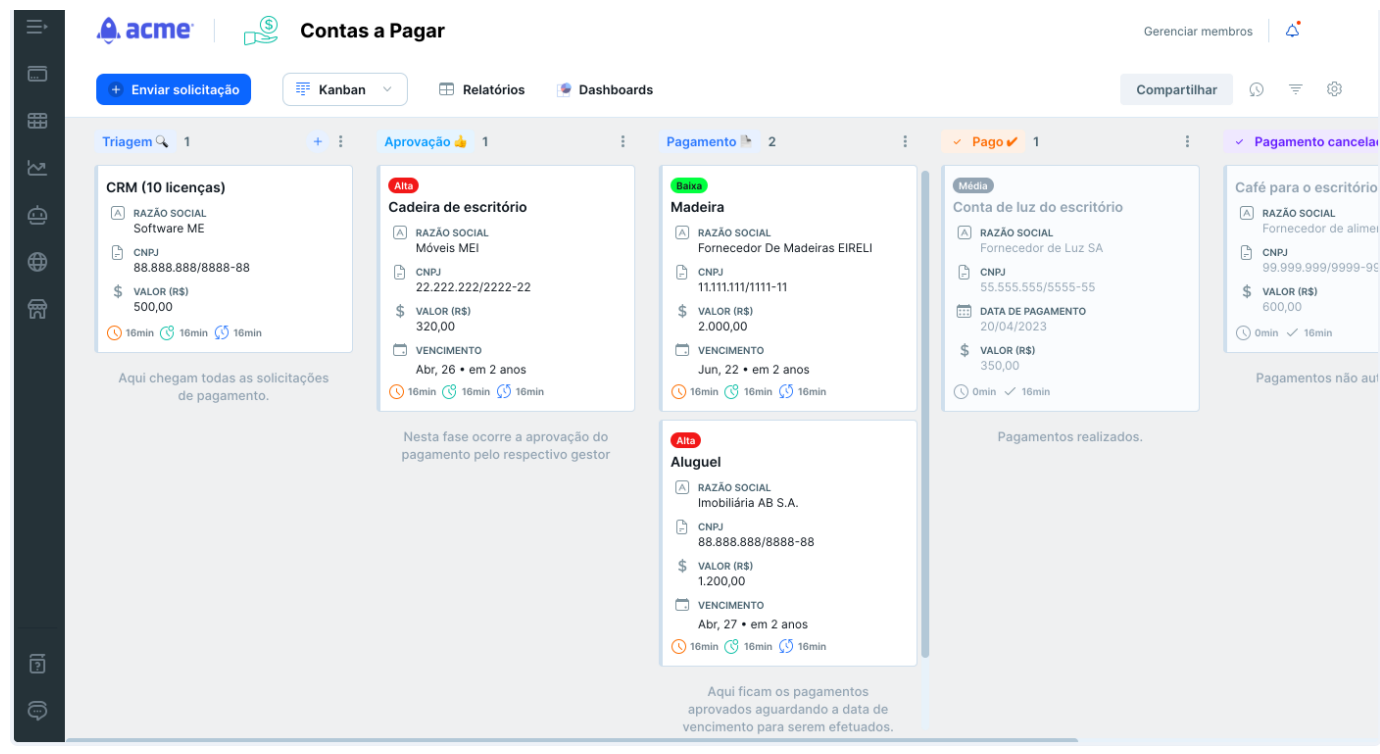

FIGURA 1 - Visualização do Pipefy. Fonte: Pipefy (2021).

O software traz as opções de relatórios e dashboards, conforme Figura 2. O Pipefy trabalha com cartões denominados pipes, no qual dentro de cada um é possível fazer anotações, inserir anexos, enviar e-mails e atualizar status. As solicitações são realizadas através de formulários públicos, que são elaborados de acordo com a necessidade da equipe, e assim padronizam o processo e melhoram a comunicação. 


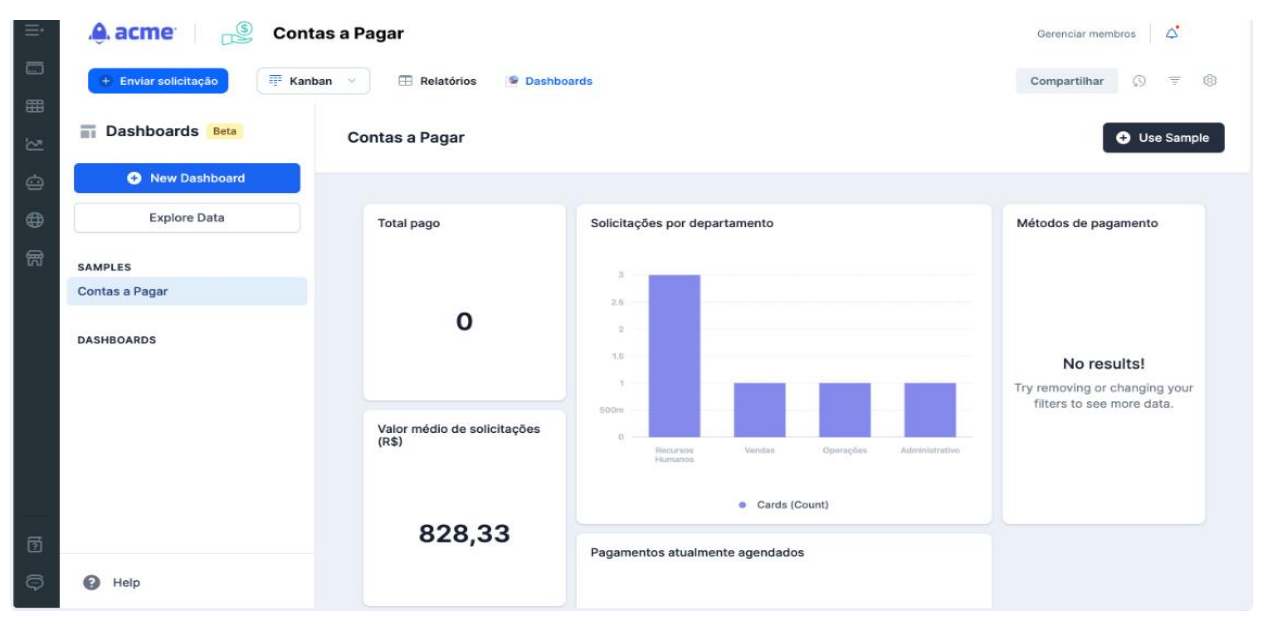

FIGURA 2 - Visualização do Dashboard. Fonte: Pipefy (2021).

\section{METODOLOGIA}

O trabalho compreendeu a realização de um estudo de casos múltiplos em três setores de uma empresa. Para Yin (2015, p. 17), o estudo de caso "investiga um fenômeno contemporâneo (o "caso") em profundidade e em seu contexto de mundo real, especialmente quando os limites entre o fenômeno e o contexto puderem não ser claramente evidentes".

A utilização de estudo de casos múltiplos possibilita a análise de mais um caso para robustecer a investigação. Como afirmam Voss, Tsikriktsis e Frohlich (2002), essa estratégia é útil para reduzir a possibilidade de enviesamento em torno de características específicas de casos únicos. Desse modo, foram selecionados três setores da empresa, Recursos Humanos, Financeiro e Comercial. Cada setor constituindo uma dinâmica de inovação no processo de trabalho e, portanto, um caso. Como estratégia de apoio à coleta e tratamento de dados, foram utilizadas as recomendações de Gioia, Corley e Hamilton (2012).

A coleta de dados teve por base entrevistas semiestruturadas com 17 colaboradores das três áreas-caso, todos participaram ativamente do processo de implantação da ferramenta de automatização de fluxo (ver detalhes na Tabela 1). A Tabela 2 aborda as questões das entrevistas semiestruturadas, que foram realizadas para entender o processo e identificar desafios enfrentados, benefícios e lições aprendidas. As informações adquiridas foram registradas em notas de campo escritas pelos pesquisadores e gravações em áudio. 
TABELA 1 - Cargo e setor dos colaboradores entrevistados.

\begin{tabular}{ccc}
\hline $\begin{array}{c}\text { Número de } \\
\text { entrevistados }\end{array}$ & Cargo & Setor \\
\hline 5 & Analista & Financeiro \\
2 & Estagiário & Financeiro \\
2 & Gerente de Área & Financeiro \\
2 & Analista & Comercial \\
1 & Gerente de Área & Comercial \\
3 & Estagiário & Recursos Humanos \\
2 & Gerente de Área & Recursos Humanos \\
\hline
\end{tabular}

Fonte: Elaborado pelos autores.

TABELA 2 - Entrevista semiestruturada.

\begin{tabular}{|c|c|}
\hline $\begin{array}{c}\text { Número de } \\
\text { questões }\end{array}$ & Questão \\
\hline 1 & Como era o processo antes da automação com Pipefy? \\
2 & Qual sua participação no processo de implantação? \\
3 & Qual a opinião do entrevistado sobre o Pipefy? \\
4 & De que forma o Pipefy afetou a rotina de trabalho? \\
5 & Quais os ganhos ao utilizar a ferramenta? \\
\hline
\end{tabular}

Fonte: Elaborado pelos autores.

Como instrumento auxiliar à coleta de dados, posteriormente às entrevistas foi confeccionado a coleta de NPS (Net Promoter Score), com uma escala de 0 a 10, a fim de avaliar o quanto cada colaborador entrevistado indica o Pipefy para amigos. De acordo com Reichheld (2003), aqueles que marcaram de 0 a 6 caracteriza-se a escala dos detratores, de 7 e 8 dos passivos e de 9 e 10 dos promotores. A Figura 3 mostra como o cálculo é realizado. 


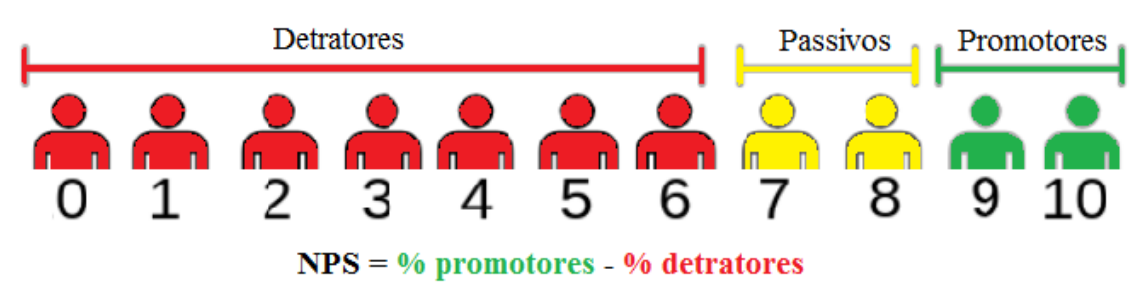

FIGURA 3 - Cálculo do NPS. Fonte: Elaborado pelos autores.

Em geral, com as notas do NPS classifica-se a empresa em quatros zonas, sendo Zona de Excelência - NPS entre 75 e 100; Zona de Qualidade - NPS entre 50 e 74; Zona de Aperfeiçoamento - NPS entre 0 e 49; Zona Crítica - NPS entre -100 e -1 (SANTOS, 2019).

Também foi confeccionado um questionário C-SAT (Customer Satisfaction Score), com uma escala de 0 a 10, com o intuito de verificar o grau de satisfação com o processo de implantação do Pipefy, conforme Figura 4.

\section{C-SAT $=\underline{\text { Classificações positivas }} \times \mathbf{~} \mathbf{1 0 0}$ \\ Classificações totais}

FIGURA 4 - Cálculo do C-SAT. Fonte: Elaborado pelos autores.

Considerou-se como classificações positivas entre 8 a 10, e acima de $70 \%$ significando um bom desempenho. No total, houve 15 respondentes do C-SAT e 15 respondentes do NPS.

Em ambos questionários foi acrescentado um campo de resposta em texto aberto para justificar a escala selecionada, e o registro de nome do respondente, de modo que foi possível retornar para coletar mais dados e esclarecer dúvidas. Esse processo foi realizado com o objetivo de poder retornar a informantes prévios para sanar dúvidas provenientes da análise (GIOIA; CORLEY; HAMILTON, 2012).

Finalmente, durante a análise dos dados foi realizado um agrupamento indutivo das respostas em conceitos de primeira e segunda ordem conforme proposto por Gioia, Corley e Hamilton (2012). Assim, os dados qualitativos puderam ser mais organizados para facilitar a análise posterior. 


\section{RESULTADOS E DISCUSSÃO}

Dado o cenário de pandemia do COVID-19, a empresa se deparou com a pressão por migrar para o regime virtual e, por consequência com maiores desafios ligados a um fluxo de trabalho eficaz. Desse modo, buscou alternativas que auxiliassem no enfrentamento desses desafios, através de uma ferramenta que possibilitasse iteração intuitiva e gerenciamento de atividades, cuja implantação consistiu em uma inovação nos processos de trabalho.

Após a decisão da implantação do Pipefy, iniciaram as iterações para entender alguns processos no regime presencial e, assim, conseguir adaptá-los para o regime remoto, com o uso do software. Foram realizadas reuniões com os envolvidos para entender os processos e identificar oportunidades de melhoria que poderiam ser geradas com o uso da ferramenta. A partir dessas reuniões foram elaborados fluxogramas com descrição de atividades, responsabilidades e prazos definidos. Com o mapeamento dos fluxos de trabalho, iniciou-se a construção do esboço da ferramenta e posteriormente a etapa de testes.

Durante a etapa de teste foram encontrados alguns problemas, como carga de dados insuficiente, impedindo a validação das informações; ausência de etiquetas dos status das atividades, impossibilitando a visualização de prioridades entre as etapas; problemas como impossibilidade de mover os cards e atualizá-los. Depois da identificação dos erros foram feitos os ajustes e diversos testes. Por fim, ao atingir o nível satisfatório de usabilidade a migração dos processos definidos foi finalizada para permitir o uso da ferramenta.

Para uma melhor visualização do fluxo de trabalho antes e depois da utilização do software, modelou-se os Fluxogramas 1 e 2. A escolha do Financeiro para modelagem dos fluxogramas de trabalho deu-se pelo fato do setor ter sido o pioneiro na implantação da automatização com o Pipefy, já tinha se desenvolvido e coordenou a implantação nos outros setores.

No Fluxograma 1 percebe-se o quanto o processo de solicitação de compra era extenso, sem a possiblidade de acompanhamento de cada etapa. 


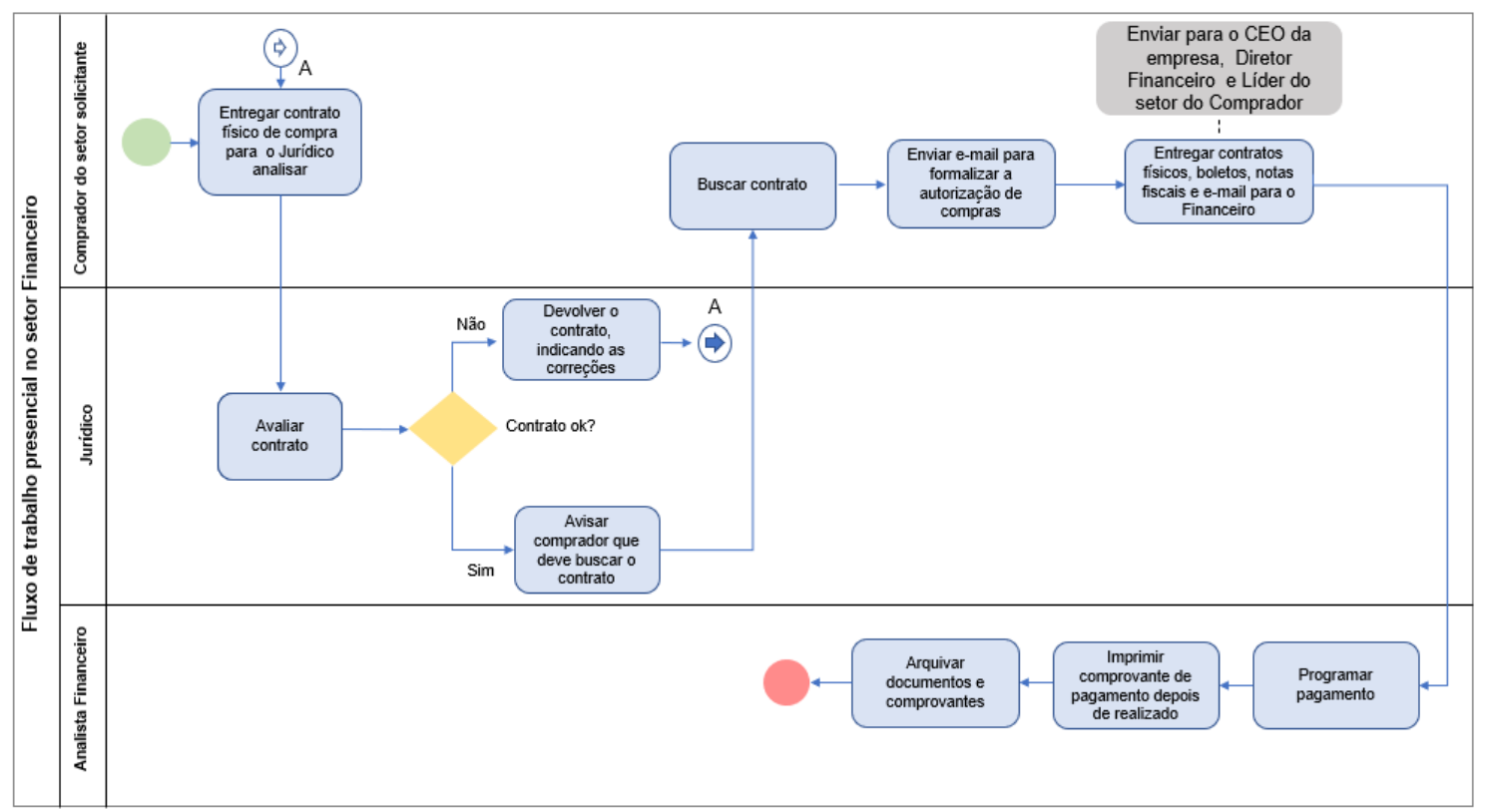

FLUXOGRAMA 1 - Fluxo de trabalho de solicitação de compra para o setor Financeiro antes da implantação do Pipefy no regime presencial. Fonte: Elaborado pelos autores.

Já no Fluxograma 2 nota-se uma diminuição das etapas e a possibilidade de acompanhamento, de forma mais automática, após a implantação do Pipefy.

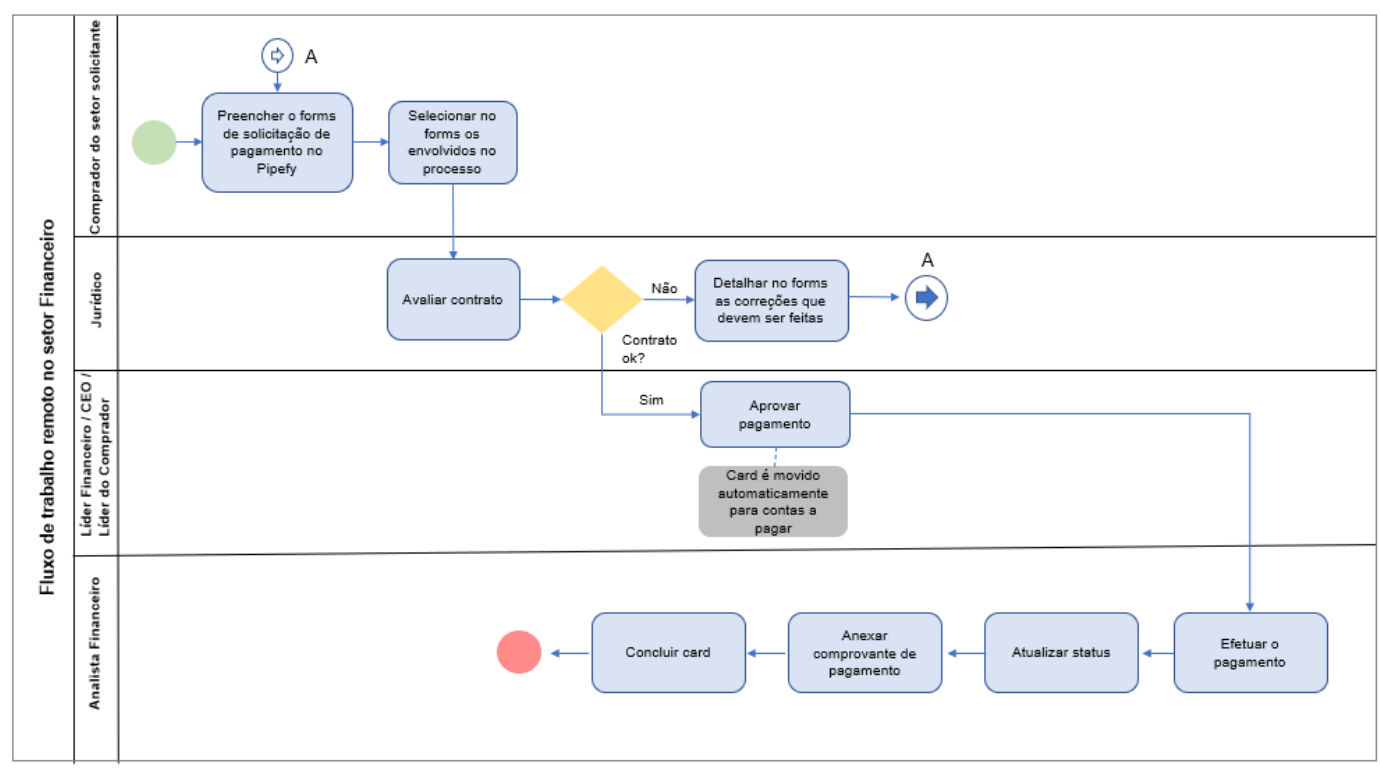

FLUXOGRAMA 2 - Fluxo de trabalho de solicitação de compra para o setor Financeiro no regime remoto após a implantação do Pipefy. Fonte: Elaborado pelos autores. 
Analisando os fluxogramas, percebe-se que houve simplificação dos fluxos, tornando a comunicação mais rápida e objetiva entre os colaboradores, fomentando seu alinhamento sobre cada etapa do processo e acesso a atualização em tempo real das demandas. A perda de dados diminuiu devido à centralização de informações em uma ferramenta, e uma melhor sequência do fluxo de trabalho ao evitar esquecimentos referentes às demandas gerenciadas informalmente ou de modo desestruturado.

Com efeito, processos bem definidos, assim como as interdependências das tarefas explicitadas são aspectos que influenciam o trabalho com equipes virtuais positivamente (ABARCA; PALOS-SANCHEZ; RUS-ARIAS, 2020; OLARIU; ALDREA, 2014). Neste sentido, a etapa inicial da implantação, voltada à compreensão dos processos para melhor descrição das atividades e definição de responsabilidades e prazos foi vista como crucial para a automatização descrita.

Após entender o processo de implantação do Pipefy por meio das entrevistas semiestruturadas, buscou-se compreender a percepção dos usuários nos setores Financeiro, Recursos Humanos e Comercial quanto à fase de implantação e utilização do software.

Ao aplicar o C-SAT com 15 colaboradores, notou-se que o nível de satisfação durante o processo em geral foi alto, $80 \%$, dado que 12 das classificações totais eram positivas, o que analisado com os demais relatos atrelam-se aos fatores citados por Abarca, Palos-Sanchez e Rus-Arias (2020) que influenciam o trabalho virtual e a comunicação dos membros da equipe, especificamente: o conhecimento da ferramenta e a simplificação do processo.

No setor de Recursos Humanos, a ferramenta o Pipefy foi completamente implantada. Já no setor Financeiro, à época do estudo alguns processos estavam passando por alterações ao uso. Vale ressaltar que com os dados obtidos pelo NPS a maioria dos colaboradores recomendam o Pipefy, sendo os promotores correspondentes a $73 \%$ dos pesquisados e os detratores a $0 \%$, conforme a Figura 3 , o resultado foi $73 \%$, esse considerado zona de qualidade. Isso é devido aos motivos citados por Cardoso (2019) e Oliveira (2020): software de apoio à gestão com interface intuitiva, automação de fluxos de trabalho efetivo com abordagens de apoio ao planejamento da equipe em horizontes de tempo. Na Tabela 3 é evidenciado as respostas das escalas, para comprovação dos cálculos do NPS e C-SAT. 
TABELA 3 - Resultados das escalas do C-SAT e NPS respectivamente.

\begin{tabular}{|c|c|}
\hline $\begin{array}{l}\text { Em uma escala de } 0 \\
\text { a } 10 \text { qual o seu nível } \\
\text { de satisfação em } \\
\text { relação ao processo } \\
\text { de implantação da } \\
\text { ferramenta Pipefy? }\end{array}$ & $\begin{array}{c}\text { Em uma escala } \\
\text { de } 0 \text { a } 10 \\
\text { quanto você } \\
\text { recomendaria o } \\
\text { Pipefy? }\end{array}$ \\
\hline 8 & 9 \\
\hline 10 & 10 \\
\hline 10 & 10 \\
\hline 9 & 9 \\
\hline 9 & 9 \\
\hline 9 & 9 \\
\hline 9 & 9 \\
\hline 9 & 9 \\
\hline 10 & 10 \\
\hline 9 & 10 \\
\hline 7 & 8 \\
\hline 8 & 8 \\
\hline 4 & 7 \\
\hline 6 & 8 \\
\hline 10 & 10 \\
\hline
\end{tabular}

Fonte: Elaborado pelos autores.

Porém, no Comercial, a adesão dos envolvidos ao processo foi menor, afirmando que a quantidade de passos que foram incluídos nas rotinas não era benéfica ao fluxo de trabalho, e que os canais de comunicação anteriores ao software, isto é, chat e e-mail, eram suficientes e relevantes para um fluxo eficaz, justificando as notas 4 e 6 nos resultados do C-SAT.

Além dos problemas durante o processo de implantação da ferramenta Pipefy, o maior desafio foi a dificuldade de aderência dos colaboradores ao processo, relacionada ao processo de implantação oneroso, que conteve diversas iterações e ajustes. Além disso, o período de adaptação foi desafiador. Mesmo que seja uma ferramenta que os colaboradores não tiveram grandes dificuldades de uso, houve um período para que se acostumassem com a migração e centralização das informações, já que o habitual era uma comunicação informal ou por e-mail.

Em relação às lições aprendidas, identifica-se pontos como: embora a plataforma seja vendida como de fácil usabilidade, a lógica de estruturação de fluxos não é simples de configurar, gerando diversas etapas de testes. Assim, as empresas devem se preparar para isso, dando atenção ao mapeamento e análise de processos prévio visto que mesmo softwares intuitivos podem apresentar alguma dificuldade percebida para implantação e rotinização do 
uso. Expandir a automatização para alguns processos/setores pode ser mais difícil do que em outros ou até inviável, como no caso do setor Comercial em comparação com o Financeiro, talvez pelo primeiro ser mais habituado à desestruturação e informalidade.

\section{CONCLUSÃO}

A presente pesquisa buscou descrever o processo de implantação de ferramenta voltada para automatização de processos de trabalho em home office durante a pandemia de COVID19. Com a proposta de analisar três setores de uma empresa, foram detectados a diminuição da perda de dados, redução de erros e melhoria na comunicação, como principais fatores de sucesso ocasionados após a implantação do Pipefy.

O processo de implantação do software passou por inúmeras iterações que resultavam em alterações do uso da ferramenta ou a definição de que o software não atendia a demanda de trabalho de alguns colaboradores. Assim, o processo de desenho dos fluxos de trabalho presencial para posterior migração para o trabalho virtual é fator crucial para inicialização da implantação, pois requer processos bem definidos, com atividades, prazos e responsabilidades descritos.

Para minimizar conflitos com a utilização do software e promover adaptabilidade no regime remoto, a empresa disponibilizou treinamentos e realizou reuniões para sanar as dúvidas e dificuldades. Todavia, registraram-se insatisfações quanto à utilidade do Pipefy pelo Comercial, ligadas ao desgaste de registro de informações e movimentações em mais uma ferramenta, dado que o setor via que e-mails e chats eram efetivos/suficientes para o trabalho realizado.

Portanto, ao propor a aplicação de uma nova ferramenta é importante primeiramente avaliar se cada grupo de usuários veem valor e se tem uma necessidade de que justifique a implantação. A empresa não analisou esse item, e apenas depois da implantação percebeu que para o Comercial o Pipefy não foi útil e que os recursos já usados eram satisfatórios.

Como abordam Abarca, Palos-Sanchez e Rus-Arias (2020), a comunicação da equipe influencia o trabalho com equipes virtuais. Assim, percebe-se que a empresa, ao disponibilizar canais de conversação desenvolveu aspectos de confiança e liderança das equipes, possibilitando que, na ausência de compartilhamento do mesmo espaço físico entre os colaboradores devido ao trabalho remoto, a comunicação organizacional não fosse prejudicada. 


\section{REFERÊNCIAS}

ABARCA, V. M. G.; PALOS-SANCHEZ, P. R.; RUS-ARIAS, E. Working in virtual teams: a systematic literature review and a bibliometric analysis. IEEE Access, v. 8, p. 168923-168940, 2020.

CARDOSO, D. M. O uso do scrum no desenvolvimento de um sistema de comunicação instantânea. 2019. Trabalho de Conclusão de Curso (Bacharelado em Sistemas de Informação) - Instituto Federal Goiano, Ceres, 2019.

GIOIA, D. A.; CORLEY, K. G.; HAMILTON, A. L. Seeking qualitative rigor in inductive research: notes on the Gioia methodology. Organizational research methods, v. 16, n. 1, p. 15-31, 2012.

GREEF, A. C. Gerenciamento de processos de negócio e workflow: estado da arte e delineamento conceitual. Revista E-Tech: tecnologias para competitividade industrial, v. 6, n. 1, p. 54-78, 2013.

HERTEL, G.; GEISTER, S.; KONRADT, U. Managing virtual teams: a review of current empirical research. Human Resource Management Review, v. 15, n. 1, p. 69-95, 2005.

LAGE JÚNIOR, M. Mapeamento de processos de gestão empresarial. Curitiba: Intersaberes, 2016.

MATTOS, J. R. L. Gestão da tecnologia e inovação: uma abordagem prática. São Paulo: Saraiva. 2012.

MELLO, A.; SOUZA, L. H. G. Solução simplificada para o monitoramento e controle de projetos utilizando a ferramenta trello. Boletim do Gerenciamento, v. 2, n. 2, 2018. Disponível em: https://nppg.org.br/revistas/boletimdogerenciamento/article/view/35. Acesso em: 17 de maio de 2021.

OLARIU, C.; ALDEA, C. C. Managing processes for virtual teams-a BPM approach. Procedia-Social and Behavioral Sciences, v. 109, p. 380-384, 2014.

OLIVEIRA, M. Aplicativo para workflow: gerenciamento de processos e fluxos de trabalho. 2020. Disponível em: https://www.pipefy.com/pt-br/blog/aplicativo-workflow/. Acesso em: 16 de maio de 2021.

PÁDUA, S. I. D.; BISPO, C. A. F. Sistema de gerenciamento de workflow: um overview e um estudo de caso. In: ENCONTRO NACIONAL DE ENGENHARIA DE PRODUÇÃO, 23., 2003, Ouro Preto. Anais [...]. Ouro Preto: UFMG, 2003.

POLETE, A. C. A.; MURITIBA, S. N.; MURITIBA, P. M.; DOMINGUES, L. M. Trabalho em equipes virtuais: efeitos da maturidade da equipe sobre a eficácia do trabalho em projetos virtuais. In: ENCONTRO DA ANPAD, 36., 2012, Rio de Janeiro. Anais [...]. Rio de Janeiro: Anpad, 2012.

REICHHELD, F. One number you need to grow. Harvard Business Review, Dec., 2003. Disponível em: https://hbr.org/2003/12/the-one-number-you-need-togrow. Acesso em: 26 jul. 2021.

RIZZI, M. Template grátis de processo de compras. Disponível em: https://www.pipefy.com/ptbr/templates/pedido-compras/. Acesso em: 16 de maio de 2021.

SÁ, M. A.; SILVA, A. E. B.; OLIVEIRA, G. F.; SILVEIRA, J. A. O Método getting things done (GTD) e as ferramentas de gerenciamento de tempo e produtividade. Navus-Revista de Gestão e Tecnologia, v. 8, n. 1, p. 72-87, 2017.

SANTOS, T. N. O serviço de ouvidoria através da pesquisa de satisfação e lealdade do cliente. Revista Científica da Associação Brasileira de Ouvidores/Ombudsman, v. 2, n. 2, p.159, 2019.

SOUZA, S. M. Estendendo ambientes de suporte a trabalho cooperativo com base no conceito de workflow. 2003. Dissertação (Mestrado) - Universidade Estadual de Campinas, Instituto de Matemática, Estatística e Computação Cientifica, Campinas, 2003. 
SUGAHARA, C. R.; JANNUZZI, C. A. S. C; FALSARELLA, O. M. Gestão do fluxo de informação em ambiente organizacional. Ciencias de la Información, v. 46, n. 2, p. 33-48, maio-agosto 2015.

TOBBERT, P. S.; ZUCKER, L. G. A Institucionalização da teoria Industrial, In: CLEGG, S.R.; HARDY, C.; NORD, W.R.; CALDAS, M.; FACHIN, R,; FISCHER, T. (Ed.). Handbook de estudos organizacionais: modelos de análises e novas questões em estudos organizacionais. São Paulo: Atlas.1999. p. 196-218.

TOWNSEND, A. M.; DEMARIE, S, M.; HENDRICKISON, A, R. Virtual teams: technology and the workplace of Ihe future. Academy of Management Executive. n. 12, p. 12 - 19, 1998.

VALENTIM, M. L. P. ; ZWARETCH. Comunicação organizacional/comunicação informacional no processo de inteligência competitiva organizacional. In: VALENTIM, M. L. P. (Org.). Informação, conhecimento e inteligência organizacional. Marília: FUNDEPE, 2006. v.5, p. 45-59.

VOSS, C.; TSIKRIKTSIS, N.; FROHLICH, M. Case research in operations management. International Journal of Operations \& Production Management, v.22, n.2, p.195-219, 2002.

WILDAUER, E. W. Mapeamento de processos: conceitos, técnicas e ferramentas. Curitiba: Intersaberes, 2015.

YIN, R. K. Estudo de caso: planejamento e métodos. 5. ed. Porto Alegre: Bookman, 2015.

ZORGIOS, Y.; KARAKOSTAS, B. Engineering service oriented systems: a model driven aproach. Hershey: IGI Global, 2008. 